\title{
The variance of $d^{\prime}$ estimates obtained in yes-no and two-interval forced choice procedures
}

\author{
WALT JESTEADT \\ Boys Town National Research Hospital, Omaha, Nebraska
}

\begin{abstract}
In studies of detection and discrimination, data are often obtained in the form of a $2 \times 2$ matrix and then converted to an estimate of $d^{\prime}$, based on the assumptions that the underlying decision distributions are Gaussian and equal in variance. The statistical properties of the estimate of $d^{\prime}, \hat{d}^{\prime}$, are well understood for data obtained using the yes-no procedure, but less effort has been devoted to the more commonly used two-interval forced choice (2IFC) procedure. The variance associated with $\hat{d}^{\prime}$ is a function of true $d^{\prime}$ in both procedures, but for small values of true $d^{\prime}$, the variance of $\hat{d}^{\prime}$ obtained using the 2IFC procedure is predicted to be less than the variance of $\hat{d}^{\prime}$ obtained using yes-no; for large values of true $d^{\prime}$, the variance of $\hat{d}^{\prime}$ obtained using the 2IFC procedure is predicted to be greater than the variance of $\hat{d}^{\prime}$ from yes-no. These results follow from standard assumptions about the relationship between the two procedures. The present paper reviews the statistical properties of $\hat{d}^{\prime}$ obtained using the two standard procedures and compares estimates of the variance of $\hat{d}^{\prime}$ as a function of true $d^{\prime}$ with the variance observed in values of $\hat{d}^{\prime}$ obtained with a 2IFC procedure.
\end{abstract}

The $d^{\prime}$ statistic describes the distance between two decision distributions in terms of their common standard deviation and is widely used as a measure of sensitivity in studies of detection and discrimination (Green \& Swets, 1966; Macmillan \& Creelman, 1991; Tanner \& Birdsall, 1958; Wickens, 2001). The two paradigms that are most frequently used to obtain estimates of $d^{\prime}$ are the yes-no and two-interval forced choice (2IFC) procedures. In the first of these, a single stimulus is presented, and the observer is asked to indicate whether or not it includes the signal. In the second, two stimuli are presented in random order, and the observer is asked to indicate which of the two stimuli includes the signal. In both cases, the results can be summarized by a $2 \times 2$ matrix showing the two types of trials (signal presented or not presented; signal presented in the first observation interval or the second) and the frequency or proportion of the observer's two responses given each type of trial. If the two types of stimuli are assumed to result in distributions of sensations that are normal with equal variance, then an estimate of the true $d^{\prime}, \hat{d}^{\prime}$, can be obtained using just two cells of the $2 \times 2$ matrix.

For a number of reasons, it is important to know the expected variance of $\hat{d}^{\prime}$ as a function of $d^{\prime}$ and of the number of trials on which $\hat{d}^{\prime}$ is based. An estimate of the vari-

This work was supported by Grant DC 006648 from the National Institutes of Health. Neil Macmillan pointed out the work by Bi, Ennis, and O'Mahony (1997) and the lack of agreement between that work and the approach to estimating $\hat{\mathrm{V}}_{d^{\prime}}$ described by Macmillan and Creelman. This work benefited from data analysis software written by Hongyang Tan and Thomas Creutz and from helpful comments by anonymous reviewers. Correspondence concerning this article should be addressed to W. Jesteadt, Boys Town National Research Hospital, 555 N. 30th Street, Omaha, NE 68131. ance is required to generate confidence intervals or to construct direct tests of the significance of the difference in two values of $\hat{d}^{\prime}$. An estimate of the variance is also necessary for assignment of appropriate weights to individual data points when using maximum likelihood methods to fit psychometric functions (Dai, 1995).

Because values of $\hat{d}^{\prime}$ obtained from $2 \times 2$ matrices are discrete rather than continuous, the sampling distribution of $\hat{d}^{\prime}$ cannot be described by a single equation. Gourevitch and Galanter (1967) derived an approximation of the variance of $\hat{d}^{\prime}$ that is accurate given a sufficiently large number of trials. Miller (1996) has computed exact values of the mean and variance of $\hat{d}^{\prime}$ as a function of true $d^{\prime}$ and the number of trials and has compared these values to the Gourevitch and Galanter approximation. Kadlec (1999) recently reported Monte Carlo simulations of the mean and variance of $\hat{d}^{\prime}$ as a function of true $d^{\prime}$, number of trials, and criterion point. All of these articles dealt only with the single-interval yes-no paradigm, a procedure that has not been used frequently in recent sensory research. Macmillan and Creelman (1991, p. 274) suggested that confidence intervals for $\hat{d}^{\prime}$ obtained with 2IFC could be arrived at by reducing confidence intervals for $\hat{d}^{\prime}$ obtained with yes-no (computed using the Gourevitch and Galanter approximation) by a factor of $\sqrt{2}$. Approximations of the variance of $\hat{d}^{\prime}$ in 2 IFC and other multiple-interval procedures provided by $\mathrm{Bi}$, Ennis, and O'Mahony (1997) suggest that the approach proposed by Macmillan and Creelman is correct when true $d^{\prime}$ is small, but not when true $d^{\prime}$ is greater than about 2.5. The approximation formula derived by $\mathrm{Bi}$ et al. is based on assumptions identical to those used by Gourevitch and Galanter. The Bi et al. formula for 2IFC, however, yields values for the variance of $\hat{d}^{\prime}$ that increase rapidly with true 
$d^{\prime}$, becoming much larger than the Gourevitch and Galanter values for $d^{\prime}>3$. Bi et al. did not include the yes-no procedure in their study and did not comment on the relationship between their variance estimates and those obtained by Gourevitch and Galanter and others for yes-no.

The purposes of the present article are to develop estimates of the variance of $\hat{d}^{\prime}$ as a function of true $d^{\prime}$ for the yes-no and 2IFC procedures, to account for the pattern of differences in these estimates as a function of procedure, and to compare the theoretical estimates of the variance of $\hat{d}^{\prime}$ with observed variances obtained in a study in which repeated measures of $d^{\prime}$ were obtained with the same observers. Estimates of the mean and variance of $\hat{d}^{\prime}$ also have been computed using the approach described by Miller (1996), which eliminates the need for Monte Carlo simulations. Miller's approach takes into account the correction for perfect performance used in the computation of $\hat{d}^{\prime}$, and in this article the analysis presented by Miller has been extended to provide a comparison of the two most common corrections (Hautus, 1995) for both yes-no and 2IFC.

\section{The Variance of $d^{\prime}$ in the Yes-No Procedure}

An example of a $2 \times 2$ matrix showing the observer's responses in a typical detection task using a yes-no procedure is shown in Table 1. If the noise alone trials and signal + noise trials are assumed to result in distributions of sensations that are normal with equal variance, then estimates of $d^{\prime}$ and of the response criterion $\beta$ can be obtained using the relations illustrated in Figure 1. The response frequencies in Table 1 are converted to proportions that correspond to areas under the normal curve, and the difference between the $z$ scores associated with those areas provides an estimate of $d^{\prime}$. The ratio of the heights of the normal curves (the ordinate of the signal+ noise distribution divided by the ordinate of the noise alone distribution) provides an estimate of $\beta$. The variance of $\hat{d}^{\prime}$ is a joint function of the binomial sampling distributions for the two underlying proportions. Basing their work on the assumption that the number of trials is sufficiently large that those binomial distributions can be approximated by normal distributions, Gourevitch and Galanter (1967) derived an estimate of the variance of $\hat{d}^{\prime}$, $\hat{\mathrm{V}}_{d^{\prime}}{ }^{1}$ for the yes-no paradigm, as shown in Equation 1 at the bottom of the page. In this equation, $H$ is the proportion of hits (the number of "yes" responses in signal+noise trials divided by $N_{\mathrm{s}+\mathrm{n}}$, the number of signal+noise trials); $1-H$ is the proportion of misses; $F$ is the proportion of false alarms (the number of "yes" responses in noise alone trials divided by $N_{\text {na }}$, the number of noise alone trials); ord $z_{1-H}$ is the height of the normal curve representing signal+noise at the $z$ score representing the proportion of misses; and ord $z_{F}$ is the height of the normal curve representing noise alone at the $z$ score representing the proportion of false alarms.
Table 1

Example of Distribution of Responses in a Yes-No Procedure

\begin{tabular}{ccc}
\hline Trial Type & Yes & No \\
\hline Signal+noise & 38 & 12 \\
Noise alone & 5 & 45
\end{tabular}

Note-The value of $\hat{d}^{\prime}$ is obtained by converting the upper left cell (hits) and the lower left cell (false alarms) to proportions, converting the proportions to $z$ scores, and then computing $z$ (hits) $-z$ (false alarms). The value of $\hat{d}^{\prime}$ for this data set would be 1.99 .

Miller (1996) obtained the expected value of $\hat{V}_{d^{\prime}}$ for many combinations of true $d^{\prime}, N_{\mathrm{s}+\mathrm{n}}$, and $N_{\mathrm{na}}$ (for the typical case in which $N_{\mathrm{s}+\mathrm{n}}=N_{\mathrm{na}}$ ) using a method that did not require approximations based on normal distributions. He began by computing the sampling distributions of $\hat{H}$ and $\hat{F}$ for a given value of true $d^{\prime}$ and a given number of trials. He then convolved those two distributions to arrive at a distribution of all possible values of $\hat{d}^{\prime}$. In both Miller's approach and Monte Carlo simulations, it is necessary to adopt a rule to handle cases in which cells of the $2 \times 2$ matrix are zero and corresponding cells are at $100 \%$ correct. The value of $\hat{d}^{\prime}$ is undefined in those cases, because the corresponding $z$ score is infinite. Miller explored the effects of three such rules: In the first, any outcome with a zero cell is repeated until an outcome without zero cells is obtained. In the second, cells in the $2 \times 2$ matrix equal to 0 are replaced by 0.5 , and cells equal to $N_{\mathrm{s}+\mathrm{n}}$ or $N_{\mathrm{na}}$ are replaced by that value -0.5 . This correction rule, recommended by Murdock and Ogilvie (1968), is widely used and is sometimes referred to as the $1 / 2 N$ correction (Hautus, 1995). In the third rule, Miller substituted 0.0001 for 0.5 in the second rule, as a means of exploring the effect of the arbitrary choice of 0.5 as a correction. In the present study, I explore the two rules compared by Hautus, the $1 / 2 N$ correction and a loglinear correction first used in the psychological literature by Snodgrass and Corwin (1988); for this log-linear correction, 0.5 is added to each of the four cell frequencies before proportions are computed. These correction rules are the ones used most frequently in psychophysics.

Regardless of what rule is adopted, a larger number of trials must be used to obtain stable values of $\hat{d}^{\prime}$ when the true $d^{\prime}$ is large. Miller (1996) found that the Gourevitch and Galanter (1967) approximation was excellent for $N_{\mathrm{s}+\mathrm{n}}, N_{\mathrm{na}}>100$ and $d^{\prime} \leq 1.0$. To give the reader an impression of the variances predicted by Gourevitch and Galanter and the size of the errors in those predictions, variance estimates obtained from Equation 1 and expected variance estimates computed using Miller's approach are plotted in Figures 2 and 3. The observer is assumed to be unbiased in all cases. Figure 2 shows $\hat{\mathrm{V}}_{d^{\prime}}$ as a function of $d^{\prime}$ for $N_{\mathrm{s}+\mathrm{n}}=N_{\mathrm{na}}=16,64$, and 256. Figure 3 shows $\hat{\mathrm{V}}_{d^{\prime}}$ as a function of $N_{\mathrm{s}+\mathrm{n}}=N_{\mathrm{na}}$ for $d^{\prime}=1,2$, and 3. In both cases, lines are used to represent approximations of $\hat{V}_{d^{\prime}}$ obtained using the Gourevitch and Galanter formula. Sym-

$$
V_{d^{\prime}}=H(1-H) / N_{\mathrm{s}+\mathrm{n}}\left(\operatorname{ord} z_{1-H}\right)^{2}+F(1-F) / N_{\text {na }}\left(\operatorname{ord} z_{F}\right)^{2}
$$




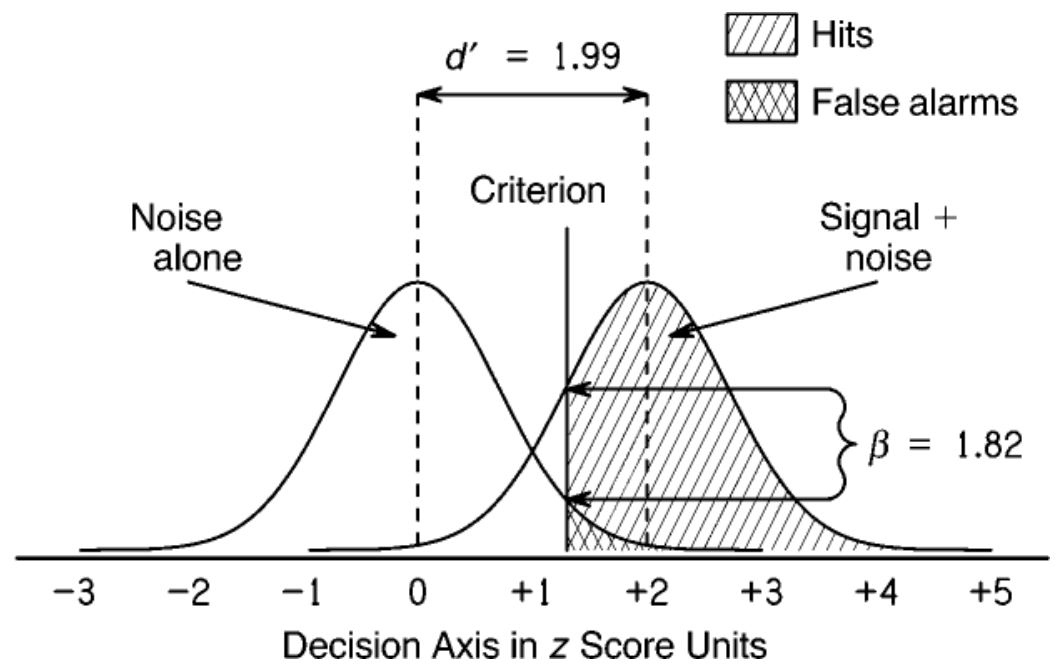

Figure 1. Illustration of standard assumptions for computation of $d^{\prime}$ and $\beta$ from $2 \times 2$ matrices for the yes-no procedure, based on the matrix given in Table 1 . In that example, the observer responded "yes" to 38 of the 50 signal+noise trials and to 5 of the noise alone trials. The corresponding areas of the signal+ noise and noise alone distributions are shaded. The $z$ scores corresponding to these areas can be used to compute $d$. The relative heights of the distributions at this criterion point are used to compute the likelihood ratio $\beta$.

bols are used to represent the expected values computed using the $1 / 2 N$ and log-linear correction rules. The values for $1 / 2 N$ agree with those reported by Miller (see his Table 2). Figure 2 shows that the large values of $\hat{V}_{d^{\prime}}$ predicted by the Gourevitch and Galanter approximation for $\hat{d}^{\prime}>2$ do not occur. Expected values computed from the sampling distributions decline at high values of $d^{\prime}$. Figure 2 also shows that the Gourevitch and Galanter approximation, which contains no correction for zero cells, is in better agreement with values of $\hat{V}_{d^{\prime}}$ obtained using the log-linear correction rather than the $1 / 2 N$ correction. Figure 3 shows that the approximation is excellent under the conditions specified by Miller but also reasonably accurate for $\hat{d}^{\prime} \leq 3.0$, if $N_{\mathrm{s}+\mathrm{n}}=N_{\mathrm{na}} \geq 32$.

The simulations reported by Kadlec (1999) indicate that values of $\hat{\mathrm{V}}_{d^{\prime}}$ increase rapidly as the criterion point moves away from the unbiased point assumed in Figures 2 and 3 . The criterion factor has been ignored here, as it was by Miller (1996), because response bias is not considered to be an important issue in data obtained with the 2IFC procedure (Green \& Swets, 1966, p. 408). ${ }^{2}$

\section{The Variance of $d^{\prime}$ in the 2IFC Procedure}

An example of a $2 \times 2$ matrix showing the observer's responses in a typical detection task using a 2IFC procedure is shown in Table 2 . The standard model for this task assumes that the decision is based on the difference between the two observations (Green \& Swets, 1966; Macmillan \& Creelman, 1991). The distance between the means of the decision distributions is therefore twice as large for a given signal level as it would be in the yes-no task. Because the decision variable is the difference between two random observations, however, the standard deviation of the decision distributions in 2IFC is larger by a factor of $\sqrt{ } 2$ than the corresponding distributions in yes-no. The net result of the increased difference between the means of the decision distributions and the increased standard deviations is that a given signal level is assumed to result in a higher proportion of correct responses in IFC than in yes-no. The expected difference in performance is illustrated in the higher percentage correct in the data in Table 2 relative to the data in Table 1. If the $2 \times 2$ matrix in Table 2 were converted to a value of $\hat{d}^{\prime}$ using the approach illustrated in Figure 1, that value would be 2.828 , a factor of $\sqrt{2}$ greater than in the yes-no paradigm. To maintain a measure of sensitivity that is independent of the experimental procedure, the convention is to obtain values of $\hat{d}^{\prime}$ in 2IFC by following the approach shown in Figure 1, then dividing the result by $\sqrt{2}$. This review has been included because the standard $\sqrt{ } 2$ correction described here plays a critical role in the relation between values of $\hat{V}_{d^{\prime}}$ for yes-no and 2IFC.

Bi et al. (1997) presented a derivation of $\hat{V}_{d^{\prime}}$ for 2 IFC for the case of equal numbers of Interval 1 and Interval 2 trials and lack of response bias, the same assumptions used above. Their approach is comparable to Gourevitch and Galanter (1967), with the addition of a standard $\sqrt{2}$ correction in the conversion from true $d^{\prime}$ to expected proportion correct. Using notation equivalent to that in Equation 1 to represent the cells in the $2 \times 2$ matrix, ${ }^{3} \mathrm{Bi}$ et al.'s findings are shown in Equation 2 at the top of the next page. They noted that the variance is proportional to 


$$
V_{d^{\prime}}=H(1-\mathrm{H}) / 2 N_{\text {Int } 1}\left(\operatorname{ord} z_{1-\mathrm{H}}\right)^{2}+F(1-F) / 2 N_{\text {Int2 }}\left(\text { ord } z_{\mathrm{F}}\right)^{2}
$$

$N$ and provided a table of values that can be divided by $N$ to arrive at variance estimates for 2IFC. Bi et al. used $N$ to refer to the total number of trials, but we have written Equation 2 in terms of $N=N_{\text {Int1 }}=N_{\text {Int2 }}$, the convention used by Gourevitch and Galanter (1967) and Miller (1996). A comparison of the variance estimates obtained with Equation 1 for yes-no and Equation 2 for 2IFC as a function of the true value of $d^{\prime}$ is shown in Figure 4, for the case of 64 trials of each type. Note that $\hat{V}_{d^{\prime}}$ for 2 IFC is less than $\hat{\mathrm{V}}_{d^{\prime}}$ for yes-no when true $d^{\prime}$ is small but that $\hat{\mathrm{V}}_{d^{\prime}}$ for 2IFC becomes larger at large values of true $d$ '.

This somewhat surprising result follows from the standard assumption that the proportion correct will be greater in 2IFC than in yes-no for any given detection or discrimination task and that an equivalent value of $\hat{d}^{\prime}$ can be obtained for 2IFC by applying a $\sqrt{2}$ "correction." Because the two equations describe variances rather than standard deviations, the difference is a factor of 2 rather than $\sqrt{2}$, and it appears as a 2 in the denominator of each term in Equation 2. This is the only difference between the two equations. How then does $\hat{V}_{d^{\prime}}$ for 2 IFC ever become larger than $\hat{\mathrm{V}}_{d^{\prime}}$ for yes-no?

Note that the variance estimates obtained using Equations 1 and 2 are a function of the proportion correct and number of trials. Assume that the level of difficulty is

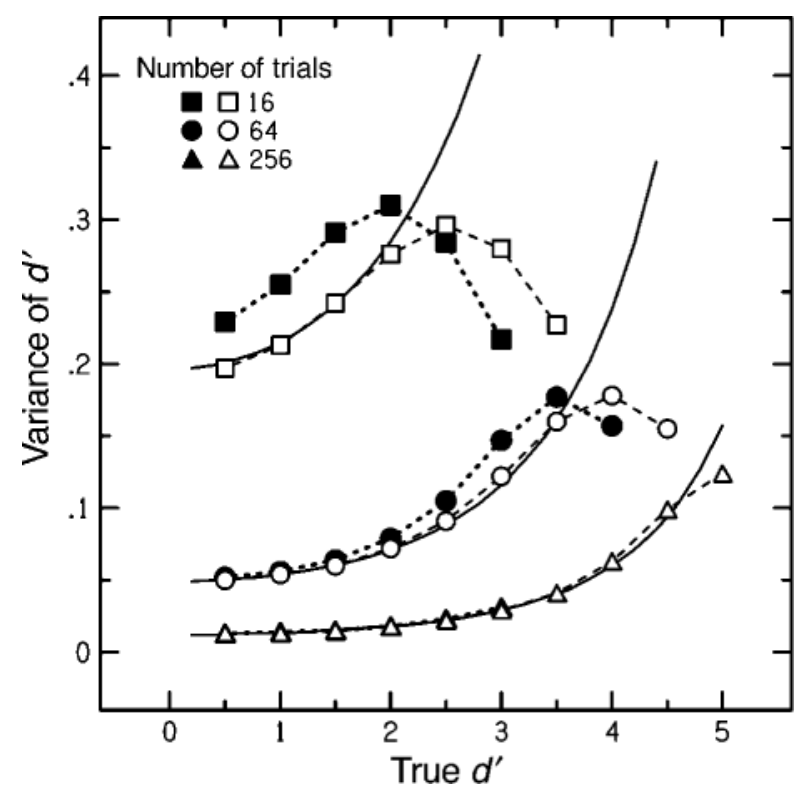

Figure 2. Values of $\hat{V}_{d^{\prime}}$ as a function of the true value of $d^{\prime}$ for the yes-no procedure. The functions show predictions of Equation 1 for $N=16,64$, or 256 trials, where $N$ is the number of noise alone trials, which equals the number of signal+noise trials. The symbols show exact values of $\hat{V}_{d^{\prime}}$ computed using the approach described by Miller (1996), with filled symbols for the $1 / 2 N$ correction and open symbols for the log-linear correction. adjusted for two discrimination tasks so that the proportion correct obtained in one task using 2IFC is equal to the proportion correct in another task using yes-no, and that the two tasks are repeated a number of times to obtain multiple values of $\hat{d}^{\prime}$ : First, the resulting value of $\hat{d}^{\prime}$ would be lower in 2IFC than in yes-no by a factor of $\sqrt{2}$. Second, the variance associated with repeated measurement of that value of $\hat{d}^{\prime}$ would be lower by a factor of 2 than the variance associated with repeated measurement of the larger $\hat{d}^{\prime}$ in yes-no. Macmillan and Creelman (1991) used this line of reasoning to argue that standard deviations for $\hat{d}^{\prime}$ obtained using 2IFC would be smaller than those obtained using yes-no by a factor of $\sqrt{ } 2$ for all values of $d$ '. The variance function they proposed is also shown in Figure 4. It agrees with Equation 2 when true $d^{\prime}$ is small.

Values of $\hat{V}_{d^{\prime}}$ for 2IFC in Figure 4 are not always lower than values of $\hat{V}_{d^{\prime}}$ for yes-no, however, because Figure 4 does not compare results obtained for equal proportions correct in the two procedures. To be consistent with previous statements of the problem, Figure 4 compares values of $\hat{V}_{d^{\prime}}$ for equal values of true $d^{\prime}$ obtained in the two procedures. A higher proportion correct is required in 2IFC than in yes-no for any given value of $d^{\prime}$. The higher proportions correct required in 2IFC for a given value of $d^{\prime}$ become an important factor as $d^{\prime}$ increases, more than compensating for the rescaling effect. Where the rescaling described by Macmillan and Creelman (1991) would result in shifting the yes-no function in Figure 4 down by a factor of 2 to arrive at the 2IFC function, the rescaling required to equate for the relation between $d^{\prime}$ and proportion correct would result in the yes-no function being shifted to the left by a factor of $\sqrt{2}$. If all of the $y$-axis values of the yes-no function are divided by 2 and all of the $x$-axis values are divided by $\sqrt{2}$, the transformed points will fall on the 2IFC function.

Over the range of proportions and number of trials for which Miller (1996) has demonstrated that Equation 1 is valid for yes-no, Equation 2 will be valid for 2IFC. For the 2IFC procedure, however, the value of $\hat{d}^{\prime}$ assigned to any given proportion correct is less by a factor of $\sqrt{ } 2$ than the corresponding value for yes-no, and the value of $\hat{V}_{d^{\prime}}$ associated with that proportion correct would be less by a full factor of 2 . The same corrections apply to expected values of $\hat{\mathrm{V}}_{d^{\prime}}$ computed using Miller's approach. ${ }^{4}$ Figure 5 shows expected values of $\hat{\mathrm{V}}_{d^{\prime}}$ for yes-no and 2 IFC when $N=64$, after each of the two corrections for zero cells has been applied. A subset of the data represented by the filled symbols can be obtained from Miller's Table 2, after the values for the 2IFC case have been shifted as described here. Because the approximation in Equation 2 fails at lower values of $d^{\prime}$ than the approximation in Equation 1, the crossover of the variance functions for 2IFC and yes-no shown in Figure 4 does not actually occur. 


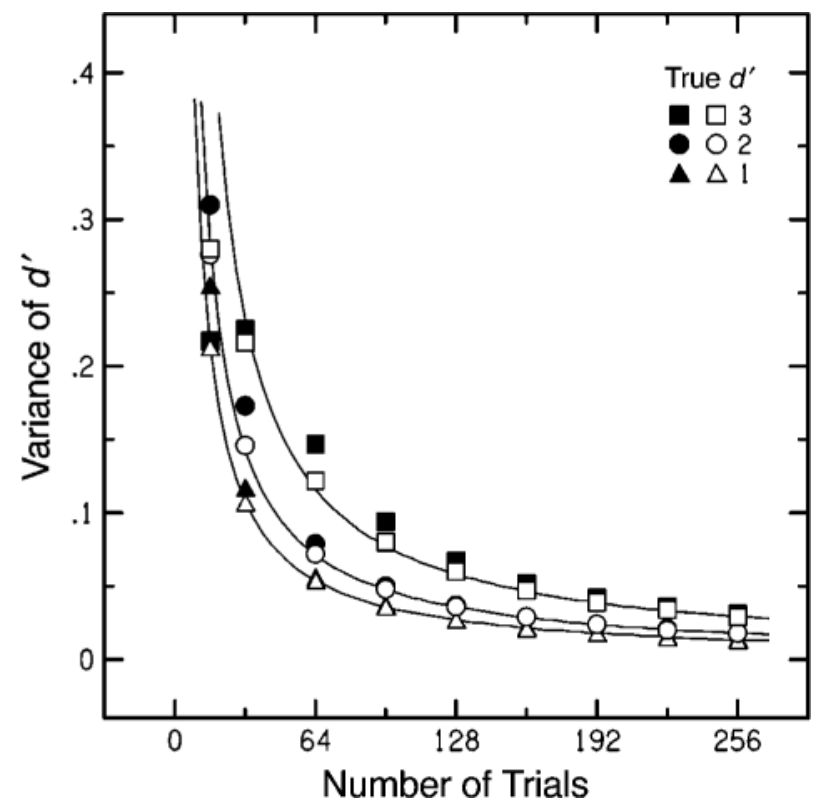

Figure 3. Values of $\hat{V}_{d^{\prime}}$ as a function of the number of noise alone or signal+noise trials for the yes-no procedure. The functions show predictions of Equation 1 for three values of $d$ '. The symbols show exact values of $\hat{V}_{d^{\prime}}$ computed using the approach described by Miller (1996), with filled symbols for the $1 / 2 N$ correction and open symbols for the log-linear correction.

\section{Observed Values of $\hat{d}^{\prime}$ and $V_{d^{\prime}}$ Obtained With a 2IFC Procedure}

Equations 1 and 2 provide estimates of the minimum values of $\hat{\mathrm{V}}_{d^{\prime}}$ that we should expect to observe in repeated measurements of $\hat{d}^{\prime}$ in the yes-no and 2IFC procedures, respectively, over the range for which the normal approximation to the binomial distribution is valid. Miller (1996) has provided a means of computing $\hat{\mathrm{V}}_{d^{\prime}}$ that does not rely on that approximation and that can easily accommodate the corrections for zero cells that are used when estimates of $d^{\prime}$ are obtained. Observed values of the variance of $d^{\prime}\left(\mathrm{V}_{d^{\prime}}\right)$ will be larger than the values of $\hat{\mathrm{V}}_{d^{\prime}}$ predicted by Miller to the extent that factors other than sampling error contribute to the variance. If those additional factors play a large role, then the theoretical relation between $\hat{V}_{d^{\prime}}$ and $\hat{d}^{\prime}$ may be irrelevant.

Many studies report mean values of $\hat{d}^{\prime}$ and standard deviations associated with those estimates, but no effort has been made to assess the contribution of the standard errors of the underlying proportions to those observed

Table 2

Example of Distribution of Responses in a 2IFC Procedure

\begin{tabular}{ccc}
\hline Trial Type & Interval 1 & Interval 2 \\
\hline Signal in Interval 1 & 46 & 4 \\
Signal in Interval 2 & 4 & 46 \\
\hline
\end{tabular}

Note-The percentage correct is higher than in the example shown in Table 1, but because the data were obtained using 2IFC, the value of $\hat{d}^{\prime}$ is the same as that for the data shown in Table $1(1.99)$. There is no response bias in the case shown. standard deviations. Figures 1 and 5 of Jesteadt, Nizami, and Schairer (2003), for example, report means and standard deviations for four repeated measurements of $\hat{d}^{\prime}$, each based on a total of 100 trials $\left(N_{\text {Int1 }}=N_{\text {Int2 }}=50\right)$ for 16 different intensity-discrimination conditions and for 7 individual subjects. In each condition, the subjects were presented with two intensities of a $2 \mathrm{kHz}$ tone of $300-\mathrm{msec}$ duration and asked to indicate which interval contained the more intense tone. The two intensities differed by only $0.1 \mathrm{~dB}$ in the most difficult condition and by $2.2 \mathrm{~dB}$ in the easiest condition, and they bracketed 50 , 70 , or $90 \mathrm{~dB}$ SPL. The conditions were designed to generate psychometric functions at the three levels. Additional details may be found in Jesteadt et al.'s study. The data for all conditions are replotted, showing mean $\mathrm{V}_{d^{\prime}}$ across the 7 subjects as a function of mean $\hat{d}^{\prime}$, in Figure 6. Predictions from Equation 2 and expected values of $\hat{\mathrm{V}}_{d^{\prime}}$ for $N=50$ also are shown. In this case, only the function for the $1 / 2 N$ correction is presented because that correction was used in the computation of the values of $\hat{d}^{\prime}$. The dotted line was obtained by multiplying points on the dashed line by a factor of 1.82 , a value chosen to maximize the fit to the data. The resulting function accounts for $63.2 \%$ of the variance in the data, whereas another fit (not shown) obtained by adding a constant to the values along the dashed line accounted for only $53.4 \%$ of the variance.

To explore the effect of carrying out the analysis illustrated in Figure 6 in standard deviation units rather than variance units, the multiplicative and additive fits were repeated after taking square roots of the observed and predicted variances. A multiplicative factor of 1.26 accounted for $62.8 \%$ of the variance, whereas the best additive constant accounted for $59.5 \%$ of the variance. The data from Jesteadt et al. (2003) are not sufficiently sensitive to distinguish between these alternatives. Although it would be helpful to have additional data of this kind, with better sampling of the higher values of $\hat{d}^{\prime}$ and a larger number of estimates per condition, Figure 6 suggests that repeated measurement of $\hat{d}^{\prime}$ results in values of $\mathrm{V}_{d^{\prime}}$ that are greater than the values predicted on the basis of binomial variance alone.

\section{Discussion}

The variance of $\hat{d}^{\prime}$ is of greatest interest for high values of $d^{\prime}$, at which point the variance begins to grow rapidly with $d^{\prime}$. As true $d^{\prime}$ increases, the correction used for zero cells in computation of $\hat{d}^{\prime}$ plays a more important role in determining both the expected mean of $\hat{d}^{\prime}$ and the expected variance. Hautus (1995) demonstrated that use of the log-linear correction resulted in an expected mean $\hat{d}^{\prime}$ that was a better approximation to true $d^{\prime}$ than would be obtained using the $1 / 2 N$ correction. Figure 2 shows that expected values of $\hat{V}_{d^{\prime}}$ obtained for the case in which $\hat{d}^{\prime}$ is computed using the log-linear correction are better represented by the Gourevitch and Galanter (1967) approximation than values of $\hat{\mathrm{V}}_{d^{\prime}}$ obtained using the $1 / 2 \mathrm{~N}$ correction. The same result holds for 2IFC. In addition, the log-linear correction for zero cells is easier to imple- 


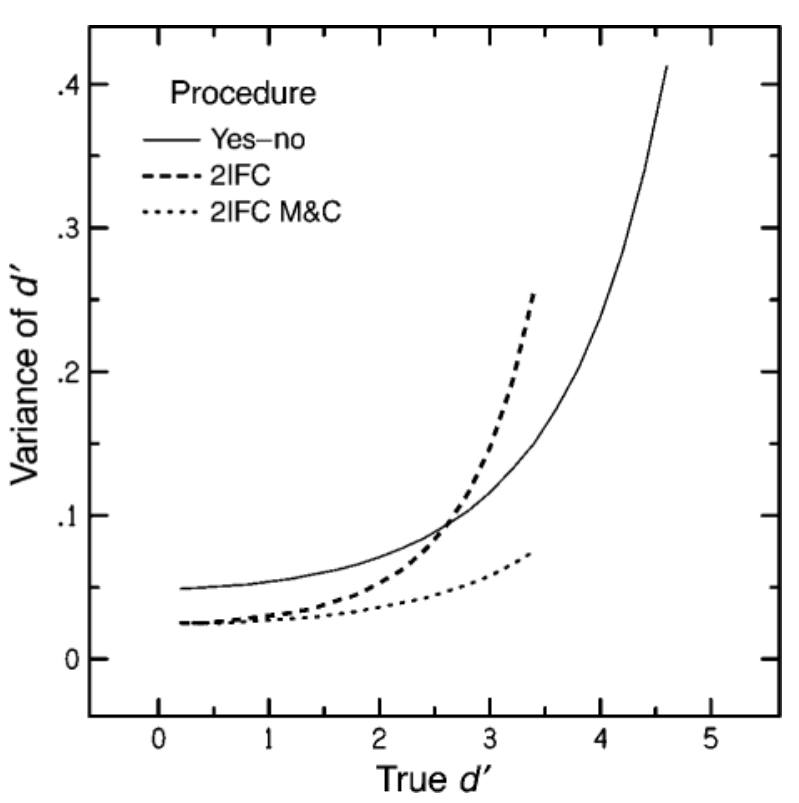

Figure 4. Values of $\hat{\nabla}_{d^{\prime}}$ as a function of the true value of $d^{\prime}$ for the yes-no and 2IFC procedures for estimates of $d^{\prime}$ based on 64 trials of each kind. The solid line shows the values of $\hat{V}_{d^{\prime}}$ for yes-no predicted by Equation 1. The dashed line shows the values of $\hat{V}_{d^{\prime}}$ for 2IFC predicted by Equation 2. The dotted line shows the values of $\hat{V}_{d^{\prime}}$ for 2 IFC predicted by a correction proposed by Macmillan and Creelman (1991).

ment. It has better properties in all respects than the more frequently used $1 / 2 N$ correction.

The results shown in Figure 4 suggest that large values of $\hat{d}^{\prime}$ are subject to significant measurement error when the number of trials is small and that these errors are substantially larger for data obtained using 2IFC. Several factors must be considered, however, that reduce the magnitude of the problem. The first is that the range of obtainable values of $\hat{d}^{\prime}$ is limited and decreases as the number of trials decreases. Computation of $\hat{d}^{\prime}$ requires that none of the cells in the $2 \times 2$ matrix be proportions of 0 or 1 , because the $z$ scores corresponding to cumulative normal probabilities of 0 or 1 are undefined. Both standard correction rules impose a greater restriction on the range of $z$ score values as the number of trials is reduced. Figure 7 shows the largest values of $\hat{d}^{\prime}$ that can be obtained with yes-no and 2IFC using either the $1 / 2 \mathrm{~N}$ or log-linear correction (the maximum values of $\hat{d}^{\prime}$ for these two corrections are nearly identical), as well as the maximum values that can be obtained using the more severe restriction that each cell in the $2 \times 2$ matrix must be $>0$ and $<1$. The functions plotted in Figures 2 and 4 were limited to the range of $d^{\prime}$ values for which corresponding estimates, $\hat{d}^{\prime}$, could be obtained using the standard corrections. This range is smaller for 2IFC than for yes-no.

The second factor, noted by Miller (1996), is that only discrete values of $\hat{d}^{\prime}$ can be obtained, and the number of possible values is more limited when true $d^{\prime}$ is large and when the number of trials of each type is small. This factor contributes to a further reduction in the value of $\hat{\mathrm{V}}_{d^{\prime}}$ in the conditions in which $\hat{\mathrm{V}}_{d^{\prime}}$ would otherwise be the greatest. The interaction of these effects results in the pattern shown in Figure 5, where the large values of $\hat{V}_{d^{\prime}}$ in 2IFC predicted by Bi et al. (1997) are not obtained using Miller's approach. For values of $N>80$, there are in fact values of true $d^{\prime}$ for which the variance in $\hat{d}^{\prime} \mathrm{ob}$ tained with 2IFC is greater than that with yes-no, but the effect never grows large because the variance associated with $\hat{d}^{\prime}$ in either procedure decreases as a function of $N$.

Given that the differences between $\hat{\mathrm{V}}_{d^{\prime}}$ for yes-no and 2IFC are completely determined by the $\sqrt{2}$ correction used in computing $\hat{d}^{\prime}$ in 2 IFC, it is ironic that the experimental data do not provide strong support for such a correction. Comparisons of yes-no and 2IFC measures of detection performance show differences less than a factor of $\sqrt{ } 2$ (e.g., Leshowitz, 1969; Markowitz \& Swets, 1967), whereas similar comparisons for intensity and frequency discrimination show differences closer to a full factor of 2 (e.g., Jesteadt \& Bilger, 1974; Pynn, Braida, \& Durlach, 1972; Viemeister, 1970). Subjects rarely base their decisions on the difference between two observations. Use of a different model of the 2IFC decision process and a different correction factor, however, would not change the amount of variability in 2IFC data in any meaningful way. The $\sqrt{ } 2$ correction rescales the data, but the mean-to-sigma ratio remains constant.

The analysis shown in Figure 6 demonstrates the value of assessing the sources of variance in repeated measures

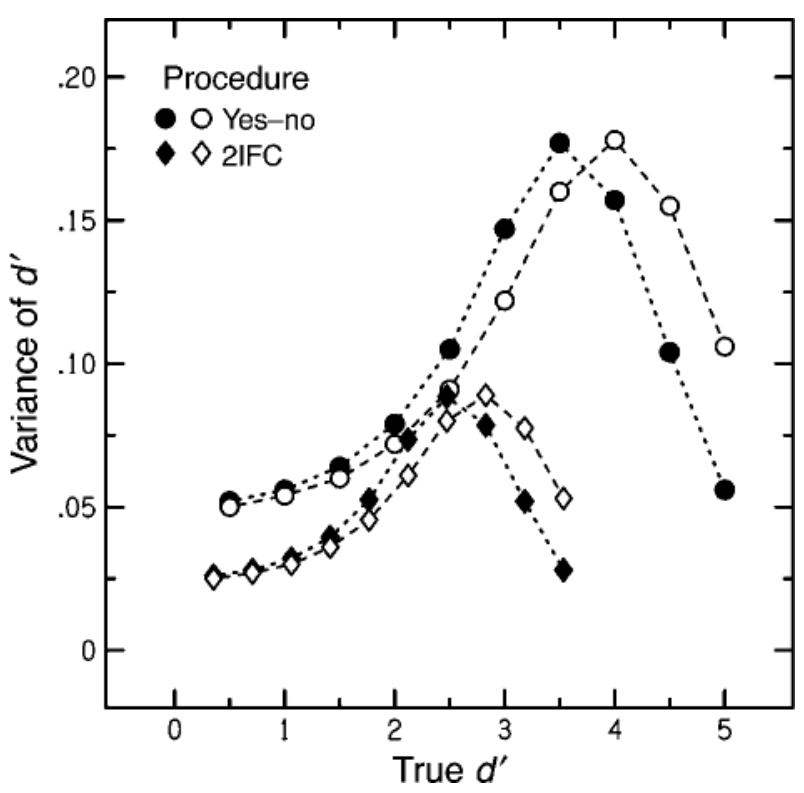

Figure 5. Values of $\hat{\nabla}_{d^{\prime}}$ as a function of the true value of $d^{\prime}$ for the yes-no and 2IFC procedures. The symbols show values of $\hat{V}_{d^{\prime}}$ computed using the approach described by Miller (1996), with filled symbols for the $1 / 2 N$ correction and open symbols for the log-linear correction, for $N=64$ trials of each kind. 


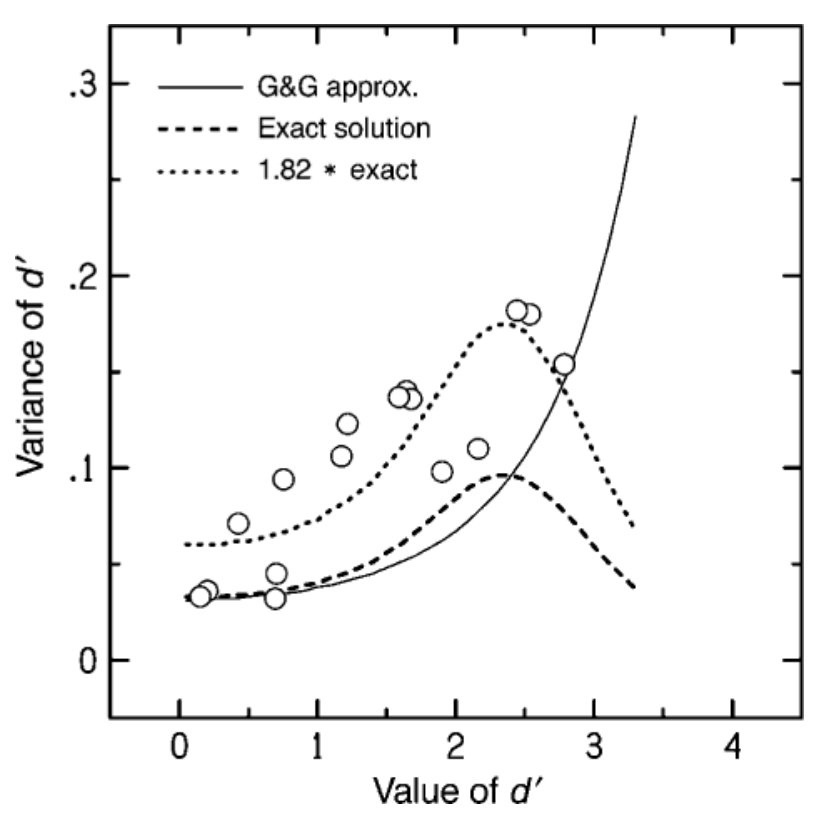

Figure 6. Observed values of $V_{d^{\prime}}$ as a function of observed values of $\hat{d}^{\prime}$ for the 2IFC procedure from Figures 1 and 5 of Jesteadt, Nizami, and Schairer (2003). The data points represent mean values of $V_{d^{\prime}}$ and $\hat{d}^{\prime}$ across 7 subjects for an intensity discrimination task in which a single fixed difference between the intensity of the two tones was used for a block of 100 trials and each condition was repeated four times. The solid line shows the prediction of Equation 2 for $N=50$. The dashed line shows exact values of $\hat{V}_{d^{\prime}}$ computed for $N=\mathbf{5 0}$ using the approach described by Miller (1996). The dotted line shows a fit to the data obtained by multiplying values on the dashed line by 1.82 .

of $\hat{d}^{\prime}$ and of understanding the source of the difference between the functions in Figure 4. The peak in the observed variance, $\mathrm{V}_{d^{\prime}}$, occurs at the point that would be predicted by functions of the type shown in Figure 5, but the values of $\mathrm{V}_{d^{\prime}}$ are higher than the predicted values by a factor of almost 2. Given the differences illustrated in Figure 4 and the literature reviewed above, it is tempting to conclude that this deviation between observed and predicted values occurred because the observers were not basing their decisions on the difference between the two observation intervals, as assumed in IIFC, but were instead performing a yes-no task, where the values of $\hat{\mathrm{V}}_{d^{\prime}}$ would be larger for given values of $\hat{d}^{\prime}$. The larger values of $\hat{V}_{d^{\prime}}$ in yes-no, however, result from the data analysis, not from increased variability on the part of the observer. If the data summarized in Figure 6 had been processed under the assumption that the observers were performing a yes-no task, the predicted values of $\hat{V}_{d^{\prime}}$ would be higher over most of the range of $\hat{d}^{\prime}$, but the observed values of $\mathrm{V}_{d^{\prime}}$ would be higher as well, and the relation between predicted and observed values would be preserved.

The observed values of $V_{d^{\prime}}$ in Figure 6 are greater than the predicted values because two sources of variance contribute to the values of $\mathrm{V}_{d^{\prime}}$ encountered in repeated measures of $\hat{d}^{\prime}$. The first is the variance associated with the binomial distributions of the proportions underlying computation of $\hat{d}^{\prime}$. Miller (1996) has provided a means of computing the expected value of that variance for any given correction for zero cells, and that approach can be extended to 2IFC as described here. The second source of variance is within the individual subject and includes variance in the coding and memory for the information, variance in criterion placement, and variance in attention. These sources are sometimes grouped under the heading of internal noise (see Jesteadt et al., 2003, for a review). Any effort to assess the degree of internal noise in terms of the variability in repeated measurements for a given subject must take into account the binomial variance inherent in making the measurements. The results in Figure 6 suggest that the internal noise could be modeled as a constant multiplier of the binomial variance. A constant multiplier of 2 would be equivalent to the $6 \mathrm{~dB}$ of multiplicative internal noise established in early detection theory studies (e.g., Green, 1960a, 1960b).

The closest parallel to the analysis illustrated in Figure 6 can be found in a later paper by Green (1990), in which he assessed the efficiency of adaptive psychophysical procedures. He first demonstrated, on the basis of the variance of binomial distributions, that an adaptive procedure that estimates the level required for $94 \%$ correct should produce results with minimum variability, and that repeated threshold estimates obtained with such a procedure should have a standard deviation that is half that of threshold estimates based on the level required

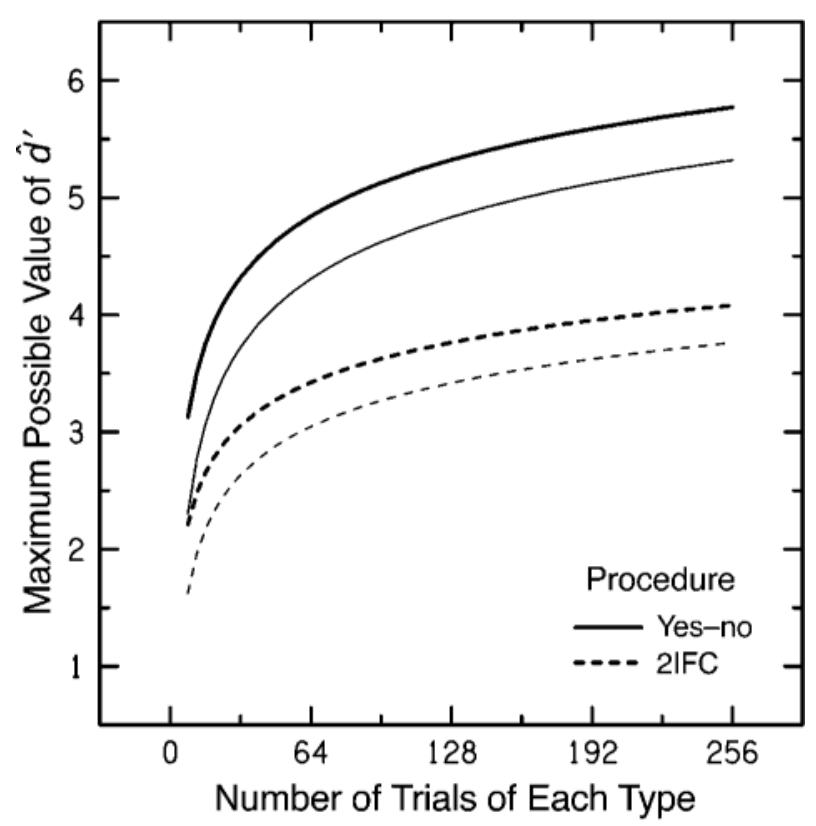

Figure 7. Maximum values of $\hat{d}^{\prime}$ that can be obtained in yes-no and 2IFC as a function of the number of trials of one type. The upper functions for yes-no and 2IFC (heavier lines) show values of $\hat{d}^{\prime}$ obtained with the $1 / 2 N$ correction for zero cells. The functions obtained with the log-linear correction are nearly identical. The lower functions (lighter lines) show the maximum values that can be obtained if data are rejected given any cells with proportions of 0 or 1. 
for $70.7 \%$ correct. He then obtained 32 estimates of threshold in a profile analysis task (Green, 1988) for each of 13 subjects using a maximum likelihood procedure designed to estimate levels corresponding to $94 \%$ and $70.7 \%$ correct. He found that the average standard deviation in threshold level across the 13 subjects was almost twice as large when threshold was measured at the lower percentage correct, as he had predicted, and he noted that this was the first such demonstration in the literature. The observed standard deviations of threshold levels were higher than predicted, however, by a factor of 1.85 at the $94 \%$ point and by a factor of 1.93 at the $70.7 \%$ point. The observed standard deviations were reduced by training. Although Green (1990) analyzed variability in threshold estimates rather than in values of $\hat{d}^{\prime}$ obtained at a fixed signal level, and although he compared standard deviations rather than variances, his pattern of results is very similar to the pattern shown in Figure 6 . For studies of individual differences in internal noise or of the effects of training, the approach described here has fewer assumptions than the analysis of psychometric functions described by Green (1990). A reanalysis of the data in Figure 6 using standard deviations rather than variances yields comparable fits to the data, but as noted above, the best-fitting ratio of observed to predicted values drops from 1.82 for variances to 1.26 for standard deviations.

The fact that observed values of $V_{d^{\prime}}$ are typically greater than the values predicted on the basis of the underlying binomial distributions means that significance tests or confidence intervals based on assumed distributions of $\hat{d}^{\prime}$ should be used with caution. Significance tests of this type are described by Gourevitch and Galanter (1967) and Bi et al. (1997). Miller (1996) discusses the related confidence intervals. To the extent that additional sources of variance are present, significance tests based on the underlying binomial distributions alone will result in underestimation of confidence intervals and a greater number of Type I errors than expected. Several important uses of predicted values of $\hat{V}_{d^{\prime}}$ remain. One is to partition the variance in repeated values of $\hat{d}^{\prime}$ into the two components described above, binomial variance and internal noise. In studies of perceptual learning of the type explored by Green (1990), it is important to be able to measure changes in variability of $\hat{d}^{\prime}$ and to factor out the effects of changes in true $d^{\prime}$ on $\mathrm{V}_{d^{\prime}}$. Analyses of the effects of internal noise on $\mathrm{V}_{d^{\prime}}$ require a better understanding of the effect of binomial variance on $\hat{V}_{d^{\prime}}$ than we have had in the past. A second application is to weight values of $\hat{d}^{\prime}$ on the basis of $\hat{\mathrm{V}}_{d^{\prime}}$ when fitting psychometric functions to values of $\hat{d}^{\prime}$ obtained at a series of stimulus levels. This is a case in which individual values of $\hat{d}^{\prime}$ are based on small numbers of trials, and the number of trials may vary from one stimulus level to the next; under these conditions, one might expect some values of $\hat{d}^{\prime}$ to be more reliable than others. The fact that values of $\hat{V}_{d^{\prime}}$ obtained using Miller's approach underestimate values of $\mathrm{V}_{d^{\prime}}$ obtained by repeated measurement is not important in constructing weights if the two variance measures are proportional, as suggested in Figure 6. Finally, studies that compare performance in different procedures and that attempt to develop more efficient procedures would also benefit from a better understanding of the factors that contribute to the variability of performance estimates.

\section{Summary and Conclusions}

Gourevitch and Galanter (1967) and Bi et al. (1997) have derived equations for $\hat{\mathrm{V}}_{d^{\prime}}$ for the yes-no and 2IFC procedures, respectively. Both derivations are based on the same standard assumptions, with the Bi et al. derivation incorporating the additional standard assumption that a $\sqrt{ } 2$ correction is necessary to equate values of $\hat{d}^{\prime}$ obtained in 2IFC procedures with those obtained in yes-no. The additional assumption for 2IFC causes $\hat{V}_{d^{\prime}}$ for 2 IFC to be less than $\hat{V}_{d^{\prime}}$ for yes-no when true $d^{\prime}$ is small but greater when true $d^{\prime}$ is large.

Values of $\mathrm{V}_{d^{\prime}}$ for 2IFC are not likely to be markedly larger than those for yes-no in actual data, because the maximum value of $\hat{d}^{\prime}$ that can be obtained for a given number of trials is smaller for 2IFC than for yes-no and because the equations derived by Gourevitch and Galanter (1967) and Bi et al. (1997) overestimate the values of $\hat{\mathrm{V}}_{d^{\prime}}$ that can occur when true $d^{\prime}$ is large.

As Miller (1996) demonstrated, values of $\hat{V}_{d^{\prime}}$ for conditions in which true $d^{\prime}$ is large are reduced by the small number of values of $\hat{d}^{\prime}$ that can be obtained and are influenced by the specific rule chosen for $\hat{d}^{\prime}$ when subjects make no errors. The log-linear rule results in values of $\hat{\mathrm{V}}_{d^{\prime}}$ that are closer to those estimated by the Gourevitch and Galanter (1967) approximation than are the values of $\hat{\mathrm{V}}_{d^{\prime}}$ obtained with the more commonly used $1 / 2 \mathrm{~N}$ rule.

Values of $\mathrm{V}_{d^{\prime}}$ observed in actual data (four replications of $\hat{d}^{\prime}$ based on a total of 100 2IFC trials per replication) were compared with values predicted by the equation derived by Bi et al. (1997) and with expected values of $\hat{\mathrm{V}}_{d^{\prime}}$ computed from the exact sampling distributions, as described by Miller (1996). The observed values of $V_{d^{\prime}}$ were greater than the expected values by about a factor of 1.8, but the form of the function relating observed $\mathrm{V}_{d^{\prime}}$ to observed $d^{\prime}$ was similar to the function predicted by Miller. This result is comparable to that reported by Green (1990) and can be attributed to multiplicative internal noise.

The differences between $\hat{V}_{d^{\prime}}$ for yes-no and 2IFC follow from a model of the decision process for 2IFC that is not supported by auditory detection and discrimination data. A change in the correction factor assumed in the model, however, would not have an impact on the relation between $\mathrm{V}_{d^{\prime}}$ and $\hat{d}^{\prime}$ observed in any given set of data.

Although estimates of $\mathrm{V}_{d^{\prime}}$ obtained from repeated measurements clearly exceed the theoretical values predicted on the basis of the underlying binomial distributions, the theoretical values have a number of applications. The range of applications is greater if it is assumed, as suggested by Figure 6, that estimates of $\mathrm{V}_{d^{\prime}}$ obtained from repeated measurements are a constant multiple of the theoretical values. 


\section{REFERENCES}

Bi, J., EnNis, D. M., \& O'MAhony, M. (1997). How to estimate and use the variance of $d^{\prime}$ from difference tests. Journal of Sensory Studies, 12, 87-104.

DAI, H. (1995). On measuring psychometric functions: A comparison of the constant-stimulus and adaptive up-down methods. Journal of the Acoustical Society of America, 98, 3135-3139.

Gourevitch, V., \& Galanter, E. (1967). A significance test for one parameter isosensitivity functions. Psychometrika, 32, 25-33.

GreEN, D. M. (1960a). Auditory detection of a noise signal. Journal of the Acoustical Society of America, 32, 121-131.

Green, D. M. (1960b). Psychoacoustics and detection theory. Journal of the Acoustical Society of America, 32, 1189-1203.

Green, D. M. (1988). Profile analysis: Auditory intensity discrimination. New York: Oxford University Press.

Green, D. M. (1990). Stimulus selection in adaptive psychophysical procedures. Journal of the Acoustical Society of America, 87, 26622674.

Green, D. M., \& Swets, J. A. (1966). Signal detection theory and psychophysics. New York: Wiley.

Hautus, M. J. (1995). Corrections for extreme proportions and their biasing effects on estimated values of $d^{\prime}$. Behavior Research Methods, Instruments, \& Computers, 27, 46-51.

Hautus, M. J., \& LeE, A. J. (1998). The dispersions of estimates of sensitivity obtained from four psychophysical procedures: Implications for experimental design. Perception \& Psychophysics, 60, 638649.

JeSteADT, W., \& Bilger, R. C. (1974). Intensity and frequency discrimination in one- and two-interval paradigms. Journal of the Acoustical Society of America, 55, 1266-1276.

JeSteadT, W., NizAmi, L., \& SCHAIRER, K. S. (2003). A measure of internal noise based on sample discrimination. Journal of the Acoustical Society of America, 114, 2147-2157.

KADLEC, H. (1999). Statistical properties of $d^{\prime}$ and $\beta$ estimates of signal detection theory. Psychological Methods, 4, 22-43.

Leshowitz, B. (1969). Comparison of ROC curves from one- and twointerval rating-scale procedures. Journal of the Acoustical Society of America, 46, 399-402.

Macmillan, N. A., \& Creelman, D. (1991). Detection theory: A user's guide. New York: Cambridge University Press.

Markowitz, J., \& Swets, J. A. (1967). Factors affecting the slope of empirical ROC curves: Comparison of binary and rating responses. Perception \& Psychophysics, 2, 91-97.

Miller, J. (1996). The sampling distribution of $d^{\prime}$. Perception \& Psychophysics, 58, 65-72.

MURDOCK, B. B., \& OGILvie, J. C. (1968). Binomial variability in shortterm memory. Psychological Bulletin, 70, 256-260.

Pynn, C. T., BraidA, L. D., \& Durlach, N. I. (1972). Intensity perception: III. Resolution in small-range identification. Journal of the Acoustical Society of America, 51, 559-566.
SNodgrass, J. G., \& Corwin, J. (1988). Pragmatics of measuring recognition memory: Applications to dementia and amnesia. Journal of Experimental Psychology: General, 117, 34-50.

TANNER, W. P., JR., \& BirdSALl, T. G. (1958). Definitions of $d^{\prime}$ and $\eta$ as psychophysical measures. Journal of the Acoustical Society of America, 30, 922-928.

VIEMEISTER, N. F. (1970). Intensity discrimination: Performance in three paradigms. Perception \& Psychophysics, 8, 417-419.

WiCKENS, T. D. (2001). Elementary signal detection theory. New York: Oxford University Press.

\section{NOTES}

1. I use the notation $\hat{V}_{d^{\prime}}$ to refer to estimates of variance of values of $\hat{d}^{\prime}$, whether those estimates are obtained using the Gourevitch and Galanter (1967) normal-curve approximation or a more accurate method described by Miller (1996). The notation $V_{d^{\prime}}$ refers to observed variance. In both cases, the variances are for $\hat{d}^{\prime}$, not true $d^{\prime}$, but the ${ }^{\wedge}$ on the $d^{\prime}$ subscript has been dropped to simplify the notation.

2. There have been no systematic studies of response bias in 2IFC, and measures of response bias are rarely reported. Kadlec's (1999) simulations suggest that response bias could be a significant source of measurement error.

3 . The labels $H, 1-H$, and $F$ were retained in Equation 2 because parallel terms do not exist for the 2IFC procedure. Thus $H$ or hits is commonly used to designate the proportion of "yes" responses given for signal+ noise, but there is no corresponding term to designate the proportion of Interval 1 responses given that the signal was presented in Interval 1. Likewise, $F$ is used to designate the proportion of "yes" responses given for noise alone, but there is no corresponding term to designate the proportion of Interval 1 responses given that the signal was presented in Interval 2. $H, 1-H$, and $F$ are used in Equation 2 to refer to the proportions in the corresponding cells of the $2 \times 2$ matrix for 2IFC. With this formulation, Equation 2 can be used to approximate $\mathrm{V}_{d^{\prime}}$ when there is a response bias in 2IFC or when $N_{\text {Int1 }} \neq N_{\text {Int2 }}$, although in those cases the approximation will not be accurate unless both $N_{\text {Int1 }}$ and $N_{\text {Int2 }}$ are large.

4. Hautus and Lee (1998) noted that Miller's (1996) equations could be corrected by a factor of 2 to obtain variance estimates for 2IFC and also presented evidence in favor of use of the log-linear correction to eliminate the possibility of cell frequencies corresponding to $0 \%$ or $100 \%$ correct. Our conclusions are thus in complete agreement. Their study was unfortunately overlooked in my review of the literature, and I became aware of it only after the present article had been accepted for publication.

(Manuscript received March 11, 2003; revision accepted for publication March 17, 2004.) 